\title{
Thermohaline mixing: a physical mechanism governing the photospheric composition of low-mass giants
}

\author{
C. Charbonnel ${ }^{1,2}$ and J.-P. Zahn ${ }^{3}$ \\ 1 Geneva Observatory, University of Geneva, chemin des Maillettes 51, 1290 Sauverny, Switzerland \\ e-mail: Corinne.Charbonnel@obs . unige.ch \\ ${ }^{2}$ Laboratoire d'Astrophysique de Toulouse et Tarbes, CNRS UMR 5572, Université Paul Sabatier Toulouse 3, 14 Av. E. Belin, \\ 31400 Toulouse, France \\ 3 LUTH, CNRS UMR 8102, Observatoire de Paris, 92195 Meudon, France \\ e-mail: Jean-Paul.Zahn@obspm.fr
}

Received 9 February 2007 / Accepted 12 March 2007

ABSTRACT

\begin{abstract}
Aims. Numerous spectroscopic observations provide compelling evidence for a non-canonical mixing process that modifies the surface abundances of $\mathrm{Li}, \mathrm{C}$ and $\mathrm{N}$ of low-mass red giants when they reach the bump in the luminosity function. Eggleton and collaborators have proposed that a molecular weight inversion created by the ${ }^{3} \mathrm{He}\left({ }^{3} \mathrm{He}, 2 \mathrm{p}\right)^{4} \mathrm{He}$ reaction may be at the origin of this mixing, and relate it to the Rayleigh-Taylor instability. We argue that one is actually dealing with a double diffusive instability referred to as thermohaline convection and we discuss its influence on the red giant branch.

Methods. We compute stellar models of various initial metallicities that include thermohaline mixing, which is treated as a diffusive process based on the prescription given originally by Ulrich for the turbulent diffusivity produced by the thermohaline instability in stellar radiation zones.

Results. Thermohaline mixing simultaneously accounts for the observed behaviour of the carbon isotopic ratio and of the abundances of $\mathrm{Li}, \mathrm{C}$ and $\mathrm{N}$ in the upper part of the red giant branch. It significantly reduces the ${ }^{3} \mathrm{He}$ production with respect to canonical evolution models as required by measurements of ${ }^{3} \mathrm{He} / \mathrm{H}$ in galactic $\mathrm{HII}$ regions.

Conclusions. Thermohaline mixing is a fundamental physical process that must be included in stellar evolution modeling.
\end{abstract}

Key words. instabilities - stars: abundances - stars: interiors - hydrodynamics

\section{Introduction}

During the first dredge-up (1dup; Iben 1965), the surface composition of low-mass red giant stars ${ }^{1}$ is modified due to dilution of the external convective stellar layers with hydrogen-processed material. The lithium and the carbon abundances as well as the carbon isotopic ratio decline, while the helium 3 and nitrogen abundances increase. The amplitude of these variations depends on the stellar mass and metallicity. This picture is nicely supported by observations.

After the 1dup the stellar convective envelope retreats (in mass) ahead of the advancing hydrogen-burning shell (HBS) that surrounds the degenerate helium core, and no further surface abundance variation is predicted by canonical stellar evolution theory ${ }^{2}$ on the RGB. However, spectroscopic observations clearly point out that some non-canonical mixing connects the convective envelope with the HBS and further modifies the surface composition of low-mass giants as soon as they reach the bump in the luminosity function. The sudden drop of the carbon isotopic ratio ${ }^{12} \mathrm{C} /{ }^{13} \mathrm{C}$ provides the most pertinent clue to this mechanism (Gilroy 1989; Gilroy \& Brown 1991; Charbonnel 1994; Charbonnel et al. 1998; Gratton et al. 2000;

${ }^{1}$ I.e., stars with initial masses below $\sim 2-2.5 M_{\odot}$ that evolve along the Red Giant Branch (RGB) to high luminosities until helium is ignited in their core under degenerate conditions.

${ }^{2}$ By this we refer to the modelling of non-rotating, non-magnetic stars, where convection is the only transport process considered.
Shetrone 2003; Recio-Blanco \& De Laverny 2007) that also modifies the abundances of lithium, carbon and nitrogen (e.g., Gratton et al. 2000). This non-canonical process appears to be universal as it affects at least $95 \%$ of the low-mass stars, whether they belong to the field, to open, or globular clusters (Charbonnel $\&$ Do Nascimento 1998). This high number satisfies the galactic requirements for the evolution of the ${ }^{3} \mathrm{He}$ abundance (Tosi 1998; Palla et al. 2000; Romano et al. 2003), since the mechanism responsible for the low values of ${ }^{12} \mathrm{C} /{ }^{13} \mathrm{C}$ is also expected to lead to the destruction of ${ }^{3} \mathrm{He}$ by a large factor in the bulk of the envelope material, as initialy suggested by Rood et al. (1984; see also Charbonnel 1995; Hogan 1995; Weiss et al. 1996).

At present, we have no firm physical model for the noncanonical RGB mixing. Parametrized approaches have been used to reproduce individual observations with the diffusive velocity treated as a free parameter (i.e. Boothroyd \& Sackmann 1999; Weiss et al. 2000). On the other hand, rotation-induced mixing has been investigated thoroughly (see references in Charbonnel \& Palacios 2004). It turns out however that meridional circulation and shear turbulence alone do not produce enough mixing to account for the surface abundance variations as required by the observations (Palacios et al. 2006).

\section{Mixing due to thermohaline convection}

Recently Eggleton et al. (2006, 2007) identified a possible cause for such non-canonical mixing, namely the molecular weight 
inversion created by the ${ }^{3} \mathrm{He}\left({ }^{3} \mathrm{He}, 2 \mathrm{p}\right){ }^{4} \mathrm{He}$ reaction in the external wing of the HBS. As pointed out already by Ulrich (1972), this nuclear reaction produces more particles per unit mass than it started from. Using their 3D hydrodynamic code to model a low-mass star at the RGB tip (Dearborn et al. 2006), Eggleton and collaborators found that such a $\mu$-profile leads to efficient mixing, of the kind required to "reconcile Big Bang and stellar nucleosynthesis (as far as ${ }^{3} \mathrm{He}$ is concerned)", as they put it.

According to them, the instability responsible for that mixing is the well-known Rayleigh-Taylor instability, a dynamical instability triggered when a layer of heavier fluid lies over lighter fluid. In stellar interiors, that instability takes the form of convective instability, which tends to render the temperature gradient adiabatic rather than to suppress the density inversion. But what first occurs in a star, as the inverse $\mu$-gradient builds up, is actually a double diffusive instability, which was discussed in the literature under the generic name of "thermohaline convection" (Stern 1960). This instability appears in various astrophysical situations, for instance when ${ }^{4} \mathrm{He}$ or $\mathrm{C}$-rich material is deposited at the surface of a star in a mass transferring binary (Stothers \& Simon 1969; Stancliffe et al. 2007). Recently it has been invoked when a star accretes heavy elements during planet formation (Vauclair 2004).

The thermohaline instability differs from the convective instability in that it involves two components, of which one, the stabilizing one (temperature) diffuses faster than the other (salt), whose stratification is unstable. It occurs in a stable stratification that satisfies the Ledoux criterion for convective stability:

$\nabla_{\mathrm{ad}}-\nabla+\left(\frac{\varphi}{\delta}\right) \nabla_{\mu}>0$

but where the molecular weight decreases with depth:

$\nabla_{\mu}:=\frac{\mathrm{d} \ln \mu}{\mathrm{d} \ln P}<0$.

We use the classical notations for $\nabla=(\partial \ln T / \partial \ln P), \varphi=$ $(\partial \ln \rho / \partial \ln \mu)_{P, T}$ and $\delta=-(\partial \ln \rho / \partial \ln T)_{P, \mu}$.

In the laboratory, the instability takes the form of "salt fingers"; since heat diffuses faster than salt, these fingers sink because they grow increasingly heavier than their environment, until they become turbulent and dissolve. In stellar interiors, the role of salt is played by a heavier species, such as helium, in a hydrogen-rich medium.

Ulrich (1972) was the first to derive a prescription for the turbulent diffusivity produced by that instability in stellar radiation zones. Through a linear analysis, and assuming perfect gas $(\varphi=\delta=1)$ he gets (his Eqs. (24) and (31))

$D_{t}=C_{t} K \frac{-\nabla_{\mu}}{\left(\nabla_{\mathrm{ad}}-\nabla\right)} \quad$ for $\nabla_{\mu}<0 ;$

$K$ is the thermal diffusivity. His non-dimensional coefficient involves the aspect ratio $\alpha$ (length/width) of the fingers:

$C_{t}=\frac{8}{3} \pi^{2} \alpha^{2}$

for the value he advocates, $\alpha=5$, this coefficient is rather large: $C_{t}=658$.

Kippenhahn et al. (1980) extended Ulrich's expression to the case of a non-perfect gas (including radiation pressure, degeneracy):

$D_{t}=C_{t} K\left(\frac{\varphi}{\delta}\right) \frac{-\nabla_{\mu}}{\left(\nabla_{\mathrm{ad}}-\nabla\right)} \quad$ for $\nabla_{\mu}<0$.

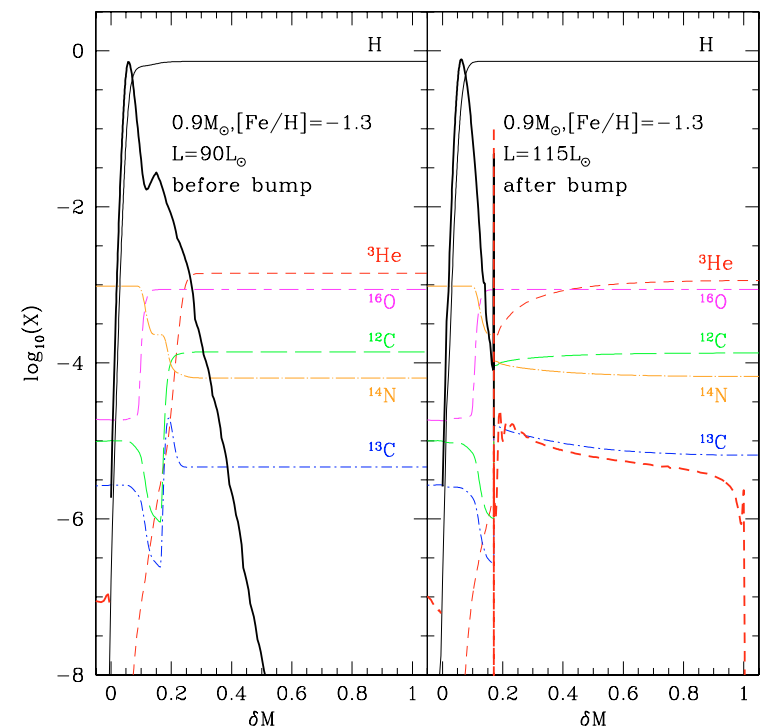

Fig. 1. Profiles of the logarithm of the mass fraction of $\mathrm{H},{ }^{3} \mathrm{He},{ }^{12} \mathrm{C},{ }^{13} \mathrm{C}$, ${ }^{14} \mathrm{~N},{ }^{16} \mathrm{O}$, and of the mean molecular weight gradient $\nabla_{\mu}=\mathrm{d} \ln \mu / \mathrm{d} \ln P$ (bold line, full when $\nabla_{\mu}>0$, dashed otherwise) as a function of the reduced mass (see text) inside a $0.9 M_{\odot},[\mathrm{Fe} / \mathrm{H}]=-1.3$ star. The left and right panels correspond to models including thermohaline mixing and located just before and after the bump. In the latter case, the thermohaline instability has just set in naturally, while in the former case it is still prevented due to the strong $\mu$-gradient left behind by the 1dup.

However instead of fingers they considered "blobs" which according to them are destroyed very rapidly: their mixing length is of the order of the blob size and this translates into a smaller value of 12 for the coefficient $C_{t}$.

It is of course disturbing that these coefficients $C_{t}$ differ by almost two orders of magnitude, depending on whom one believes; it would be most desirable to confront them with realistic 3D simulations. In the meanwhile, we favour a large value for the coefficient $C_{t}$ as advocated by Ulrich, because all experiments so far have displayed slender fingers, rather than blobs (cf. Krishnamurti 2003). Crude as it may be, this prescription has the advantage of being rooted in the physical process, unlike the admittedly ad hoc diffusivity chosen by Eggleton et al. (2007).

\section{Model calculations}

We compute three evolution models of a $0.9 M_{\odot}$ star with various initial values of $[\mathrm{Fe} / \mathrm{H}]$, namely $-1.8,-1.3$ and -0.5 , in order to compare our theoretical predictions with observations over a large range of metallicity. We use the code STAREVOL (Siess et al. 2000; Palacios et al. 2003, 2006). In the present study the transport of particles in the radiative regions is due to thermohaline mixing only. The expression we use for $D_{t}$ is that given in Eq. (5) and includes the correction for a non-perfect gas. For simplicity we assume $C_{t}=1000$, which is of the order of magnitude of the coefficient advocated by Ulrich (1972). The model at $[\mathrm{Fe} / \mathrm{H}]=-1.3$ is also computed with $C_{t}=100$ and 12 . We do not consider any additional transport process related to rotation.

Figure 1 presents the abundance profiles of selected elements and the mean molecular weight gradient $\nabla_{\mu}=\mathrm{d} \ln \mu / \mathrm{d} \ln P$, just before and after the bump in the $0.9 M_{\odot},[\mathrm{Fe} / \mathrm{H}]=-1.3$ star when $C_{t}=1000$. The abscissa is the relative mass $\delta M=$ $\left(M_{r}-M_{\text {core }}\right) /\left(M_{\text {env }}-M_{\text {core }}\right)$ defined as ranging from 0 to 1 


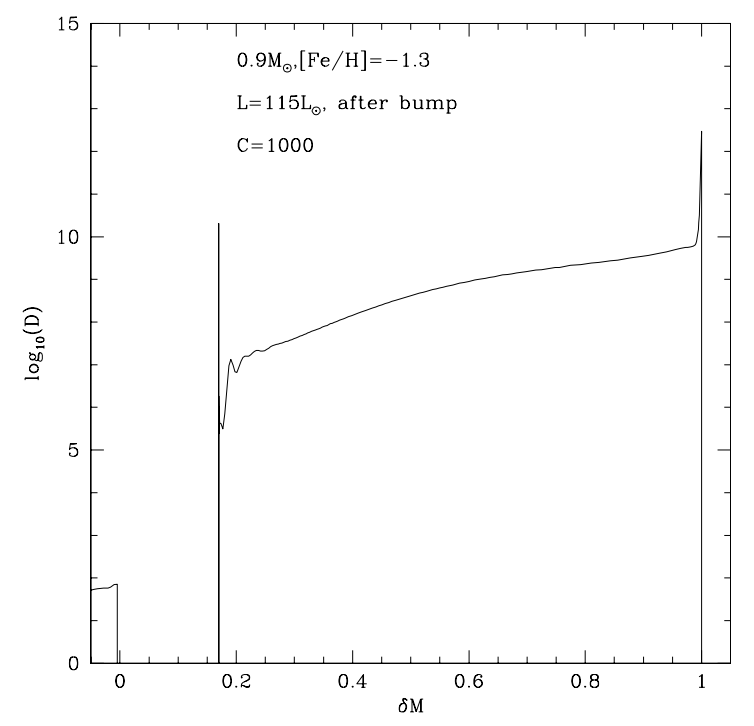

Fig. 2. Logarithm of the diffusion coefficient $D_{t}$ (assuming $C_{t}=1000$ ) inside the $0.9 M_{\odot},[\mathrm{Fe} / \mathrm{H}]=-1.3$ star just after the bump.

between the bottom of the HBS and the base of the convective envelope.

During the 1dup phase, the convective envelope homogenizes the star down to very deep regions, and builds a very steep gradient of molecular weight at the point of its maximum penetration. This corresponds to the external peak (at $\delta M \sim 0.12$ ) in the profile of $\nabla_{\mu}$ that can be seen in the left panel of Fig. 1. On the other hand the deeper peak (at $\delta M \sim 0.05$ ) corresponds to the region where $\mathrm{H}$ is efficiently depleted by nuclear reactions in the HBS. One sees that at the current luminositity $\left(L=90 L_{\odot}\right)$ both peaks are very close to each other, meaning that the star is approaching the bump. At this evolutionary point, $\nabla_{\mu}$ is positive in the whole radiative region, and the thermohaline instability cannot set in (see Eq. (2)). The profiles of the chemical elements are thus identical to those obtained in a canonical model.

When the HBS passes through the $\mu$-discontinuity left behind by the 1 dup (i.e., at the bump), H-burning occurs in a homogeneous region. The ${ }^{3} \mathrm{He}\left({ }^{3} \mathrm{He}, 2 \mathrm{p}\right){ }^{4} \mathrm{He}$ reaction slightly lowers the molecular weight in the external wing of the HBS where $\nabla_{\mu}$ becomes negative (bold dashed line in the right panel of Fig. 1), allowing the thermohaline instability to develop naturally between the ${ }^{3} \mathrm{He}$-burning region and the base of the convective envelope. Deeper inside the radiative region, $\nabla_{\mu}$ remains positive (bold full line) and no thermohaline mixing occurs. As can be seen in Fig. 1, the surface abundance of ${ }^{3} \mathrm{He},{ }^{12} \mathrm{C},{ }^{13} \mathrm{C}$, and ${ }^{14} \mathrm{~N}$ are already modified soon after the onset of thermohaline mixing. However the surface abundance of ${ }^{16} \mathrm{O}$ remains constant because the thermohaline mixing does not extend down to the very deep region where full CNO-burning operates at equilibrium.

We show in Fig. 2 the profile of the diffusion coefficient $D_{t}$ (Eq. (5), for $\left.C_{t}=1000\right)$, for the same model just after the onset of the thermohaline instability $\left(L=115 L_{\odot}\right)$. $D_{t}$ is high enough to connect the HBS and the convective envelope and to modify the surface abundances as shown below. It is two to three orders of magnitude higher than that characterizing the rotational mixing (Palacios et al. 2006).

\section{Signatures of mixing and comparison with observations}

In Fig. 3 we compare the temporal evolution of ${ }^{7} \mathrm{Li},{ }^{12} \mathrm{C} /{ }^{13} \mathrm{C}$, $[\mathrm{C} / \mathrm{Fe}]$, and $[\mathrm{N} / \mathrm{Fe}]$, obtained at the surface of our mod-

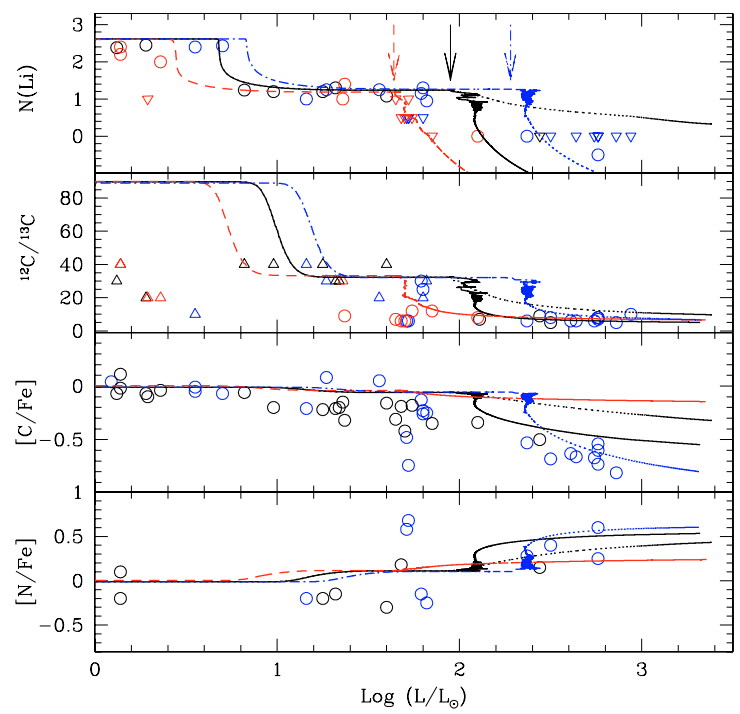

Fig. 3. Evolution of the lithium abundance, of the carbon isotopic ratio ${ }^{12} \mathrm{C} /{ }^{13} \mathrm{C}$, of $[\mathrm{C} / \mathrm{Fe}]$ and $[\mathrm{N} / \mathrm{Fe}]$ as a function of the luminosity logarithm for the $0.9 M_{\odot}$ models with $[\mathrm{Fe} / \mathrm{H}]=-1.8,-1.3$ and -0.5 (respectively dashed-dotted blue, full line black, and dashed red) computed with $C_{t}=$ 1000 in Eq. (5). The black dotted line is for the model with $[\mathrm{Fe} / \mathrm{H}]=$ -1.3 calculated with $C_{t}=100$. The arrows in the upper panel indicate the location of the bump for the three metallicities. Observational data are from Gratton et al. (2000) for field stars in the metallicity range $[\mathrm{Fe} / \mathrm{H}] \in[-2 ;-1]$. Blue symbols are for stars with measured $[\mathrm{Fe} / \mathrm{H}]$ values lower than -1.4 , black for stars with $-1.4 \leq[\mathrm{Fe} / \mathrm{H}] \leq-1.2$, and red for $[\mathrm{Fe} / \mathrm{H}]$ higher than -1.2 . Circles are actual measurements, open upward triangles are lower limits and open downward triangles are upper limits.

els, with homogeneous observational data for field stars with $-2 \leq[\mathrm{Fe} / \mathrm{H}] \leq-1$ by Gratton et al. (2000). We see that variations associated with the 1dup fit well the observational behaviour on the lower RGB. The surface abundances then stay constant until the stars reach the bump. Until that evolutionary point the predictions are identical to those of the canonical models.

Then at the luminosity of the bump (which increases for a given stellar mass when the initial metallicity decreases, as shown by the arrows) thermohaline mixing leads to a second episode of abundance variations that explains remarkably well the data ${ }^{3}$ Li is rapidly destroyed, and the carbon isotopic ratio reaches values between 5 and 7 very close to the equilibrium value. $[\mathrm{C} / \mathrm{Fe}]$ and $[\mathrm{N} / \mathrm{Fe}]$ respectively decrease and increase. These $\mathrm{C}$ and $\mathrm{N}$ variations are stronger for lower initial metallicity, as required by the observations. As already noted in Sect. 3, the surface abundances of the heavier elements $\left({ }^{16} \mathrm{O}\right.$ and ${ }^{23} \mathrm{Na}$ in particular, not shown here), which are affected by nuclear reactions much closer to the He-core, do not vary at the surface.

The models shown in Fig. 3 were computed assuming $C_{t}=1000$ in Eq. (5). We also show the predictions for the model with $[\mathrm{Fe} / \mathrm{H}]=-1.3$ computed with $C_{t}=100$. In that case the surface abundances change less rapidly and one does not reproduce the abrupt variation at the luminosity of the bump seen in the observations. Our analysis is thus compatible with the high value for the coefficient $C_{t}$ advocated by Ulrich (1972) and it excludes

3 Oscillations in stellar luminosity can be seen just above the bump. They are related to the transport of ${ }^{3} \mathrm{He}$ from the convective envelope into the outer HBS region where this element burns in favour of ${ }^{7} \mathrm{Be}$. The related nuclear energy release is large. When it happens to rival that of the pp-chains, the total luminosity increases and the star readjusts. Once the abundance of ${ }^{3} \mathrm{He}$ is low enough the oscillations vanish. 


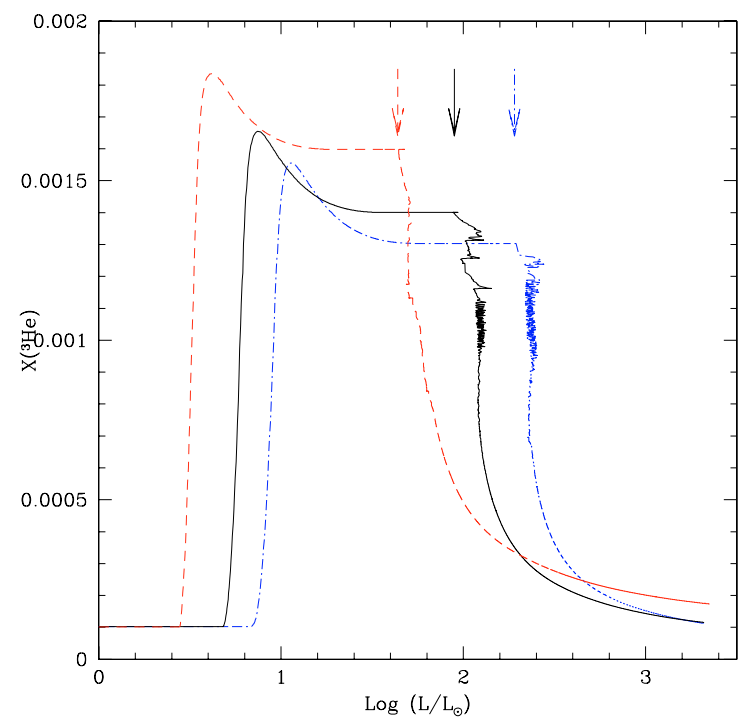

Fig. 4. Evolution of the surface abundance of ${ }^{3} \mathrm{He}$ (in mass fraction). The line symbols are as in Fig. 3.

the low value (12, not shown here) derived by Kippenhahn et al. (1980).

\section{The case of ${ }^{3} \mathrm{He}$}

On the main sequence, a ${ }^{3} \mathrm{He}$ peak builds up due to pp-reactions inside the low-mass stars (Iben 1967), and is engulfed in the stellar envelope during the $1 \mathrm{dup}$. As a consequence the surface abundance of ${ }^{3} \mathrm{He}$ strongly increases on the lower RGB as can be seen in Fig. 4 for our models at three different metalicities. Its value reaches a maximum when the whole peak is engulfed. After the 1dup the temperature at the base of the convective envelope is too low for ${ }^{3} \mathrm{He}$ to be nuclearly processed. As a result, in canonical models ${ }^{3} \mathrm{He}$ stays constant at the surface and its final value is strongly increased with respect to the initial one (this is the value before thermohaline mixing sets in at the bump).

After the bump, thermohaline mixing brings ${ }^{3} \mathrm{He}$ from the convective envelope down to the region where it is nuclearly burned. This leads to a rapid decreases of the surface abundance of this element as can be seen in Fig. 4. This confirms the early suggestion by Rood et al. (1984) that the variations of the carbon isotopic ratio and of ${ }^{3} \mathrm{He}$ are strongly connected (see also Charbonnel 1995; Eggleton et al. 2007). It is important to note that in the models presented here ${ }^{3} \mathrm{He}$ decreases by a large factor in the ejected material with respect to the canonical evolution predictions but that low-mass stars remain net producers of ${ }^{3} \mathrm{He}$ (while far much less efficient than in the canonical case). Computations for different masses and metallicities have now to be performed in order to estimate the actual contribution of lowmass stars to Galactic ${ }^{3} \mathrm{He}$ in the framework proposed here. We are confident that the corresponding ${ }^{3} \mathrm{He}$ yields will help reconcile the primordial nucleosynthesis with measurements of ${ }^{3} \mathrm{He} / \mathrm{H}$ in galactic HII regions (Balser et al. 1994; Bania et al. 2002; Charbonnel 2002).

\section{Conclusions}

It is well known that a non-canonical mixing process modifies the surface abundances of low-mass stars when they arrive at the bump in the luminosity function. Based on 3D-modeling of a low-mass star at the RGB tip, Eggleton and collaborators have proposed that the molecular inversion created by the ${ }^{3} \mathrm{He}\left({ }^{3} \mathrm{He}, 2 \mathrm{p}\right){ }^{4} \mathrm{He}$ reaction in the external wing of the HBS may be the cause of such a mixing. They ascribe this mixing to the Rayleigh-Taylor instability.

Here we argue that one is actually dealing with a double diffusive instability called thermohaline convection, which has been discussed long ago in the literature. We compute stellar models including the prescription given by Ulrich (1972) and extended to the case of a non-perfect gas for the turbulent diffusivity produced by that instability in the stellar radiative zone. The results presented here indicate that thermohaline convection simultaneously accounts for the observed behaviour of the carbon isotopic ratio and for the abundances of $\mathrm{Li}, \mathrm{C}$ and $\mathrm{N}$ in $\mathrm{RGB}$ stars at and above the bump in the luminosity function. It also avoids large ${ }^{3} \mathrm{He}$ production by low-mass stars as required by chemical evolution models of the Galaxy. It does not modify the $\mathrm{O}$ nor the Na surface abundances. In a future work the effect of thermohaline mixing in models of various masses will be presented.

Acknowledgements. We thank the referee for her/his comments that helped us improve our manuscript and Dr Eggleton for sending us his paper before publication. C.C. is supported by the Swiss National Science Foundation (FNS). We acknowledge travel support from the French Programme National de Physique Stellaire (PNPS)

\section{References}

Balser, D. A., Bania, T. M., Brockway, C. J., Rood, R. T., \& Wilson, T. L. 1994, ApJ, 430, 667

Bania, T. M., Rood, R. T., \& Balser, D. A. 2002, Nature, 415, 54

Boothroyd, A. I., \& Sackmann, I. J. 1999, ApJ, 510, 232

Charbonnel, C. 1994, A\&A, 282, 811

Charbonnel, C. 1995, ApJ, 453, L41

Charbonnel, C. 2002, Nature, 415, 27

Charbonnel, C. 2005, From lithium to uranium: Element tracers of early cosmic evolution, ed. V. Hill, P. François, F. Primas (Cambridge Univ. Press), IAU Symp., 228, 347

Charbonnel, C., \& do Nascimento, J. D. 1998, A\&A, 336, 915

Charbonnel, C., \& Palacios, A. 2004, IAU Symp., 215, 440

Charbonnel, C., Brown, J. A., \& Wallerstein, G. 1998, A\&A, 332, 204

Dearborn, D. S. P., Eggleton, P. P., \& Lattanzio, J. C. 2006, ApJ, 639, 405

Eggleton, P. P., Dearborn, D. S.P., \& Lattanzio, J. C. 2006, Science, 314, 1580

Eggleton, P. P., Dearborn, D. S. P., \& Lattanzio, J. C. 2007, private communication

Gilroy, K. K. 1989, ApJ, 347, 835

Gilroy, K. K., \& Brown, J. 1991, ApJ, 371, 578

Gratton, R. G., Sneden, C., Carretta, E., \& Bragaglia, A. 2000, A\&A, 354, 169

Hogan, C. J. 1995, ApJ, 441, L17

Iben, I. 1967, ApJ, 143, 642

Kippenhahn, R., Ruschenplatt, G., \& Thomas, H. C. 1980, A\&A, 91, 175

Krishnamurti, R. 2003, J. Fluid Mech., 483, 287

Palacios, A., Talon, S., Charbonnel, C., \& Forestini, M. 2003, A\&A, 399, 603

Palacios, A., Charbonnel, C., Talon, S., \& Siess, L. 2006, A\&A, 453, 261

Palla, F., Bachiller, R., Stanghellini, L., Tosi, M., \& Galli, D. 2000, A\&A, 355, 69

Recio-Blanco, A., \& de Laverny, P. 2007, A\&A, 461, L13

Romano, D., Tosi, M., Matteucci, F., \& Chiappini, C. 2003, MNRAS, 346, 295

Rood, R. T., Bania, T. W., \& Wilson, T. L. 1984, ApJ, 280, 629

Shetrone, M. D. 2003, in CNO in the Universe, ed. C. Charbonnel, D. Schaerer, \& G. Meynet, ASP Conf. Ser., 304, 137

Siess, L., Dufour, E., \& Forestini, M. 2000, A\&A, 358, 593

Stancliffe, R. J., Glebbeek, E., Izzard, R. G., \& Pols, O. R. 2007, A\&A, 464, L57

Stern, M. E. 1960, Tellus, 12, 172

Stothers, R., \& Simon, N. R. 1969, ApJ, 157, 673

Tosi, M. 1998, Space Sci. Rev., 84, 207

Ulrich, R. K. 1972, ApJ, 172, 165

Vauclair, S. 2004, ApJ, 605, 874

Weiss, A., Wagenhuber, J., \& Denissenkov, P. A. 1996, A\&A, 313, 581

Weiss, A., Denissenkov, P. A., \& Charbonnel, C., 2000 A\&A, 356, 181 